\title{
Höfischer Luxus oder stehendes Heer? Eine bisher unbekannte Schrift Veit Ludwig von Seckendorffs aus dem Jahr 1672 (mit Edition)"
}

von

CHRISTIAN HEINKER

Im Juli 1672 trafen sich in Leipzig die Vertreter der Stände des Obersächsischen Reichskreises ${ }^{1}$ aus aktuellem Anlass zu einem Konvent. Diese Versammlung hatte seit immerhin sieben Jahren nicht mehr stattgefunden; nun, unter dem Eindruck des Einmarsches Frankreichs in die Republik der Vereinigten Niederlande, ${ }^{2}$ wurde es (wieder einmal) ein Kriegskonvent. Überaus zäh und langwierig gestalteten sich die Verhandlungen um ein militärisches Bündnis, vor allem weil Kurbrandenburg seinen Anteil zur Aufstellung eines Reichsheeres als bereits erfüllt ansah und seine Politik zunehmend ohne Rücksicht auf das Reich und seine Bündnisverpflichtungen betrieb. Kurbrandenburg war es als erstem Reichsstand gelungen, seine Landeshoheit auf das in der Frühen Neuzeit so wichtige Feld der Wehrverfassung auszudehnen.

Andererseits gelang es Kursachsen schon seit geraumer Zeit immer weniger, seine Rolle als Kreisoberster ${ }^{3}$ auch tatsächlich auszufüllen. Die Einrichtung der Sekundoge-

* Frau Roswitha Franke vom Sächsischen Staatsarchiv - Staatsarchiv Leipzig sei an dieser Stelle für die Veröffentlichungsgenehmigung gedankt.

1 1500/1512 wurden auf den Reichstagen von Augsburg bzw. Trier zehn Reichskreise geschaffen, denen vor allem rechtliche(Vollstreckung von Reichskammergerichtsurteilen) und finanztechnische (Münzwesen) Aufgaben oblagen. Auf dem Wormser Reichstag 1521 wurde auf Basis einer Landfriedensordnung ein Reichsheerwesen geschaffen. Den Reichskreisen kamen dabei die einzelnen Kontingente des Reichsheeres zu, das im Kriegsfall aus dem ,Simplum'von 4000 Reitern und 20000 Fußknechten bestand. Diese ,Reichswehrverfassung' blieb bis in die zweite Hälfte des 17. Jahrhunderts bestehen. Der Westfälische Frieden hatte den Reichsständen das Recht auf eigene Truppen garantiert, was die Landesfürsten der größeren Territorien bald für sich nutzten. $\mathrm{Zu}$ den Reichskreisen vgl. Winfried DotZauer, Die deutschen Reichskreise in der Verfassung des alten Reiches und ihr Eigenleben. 1500-1806, Darmstadt 1989; Peter Claus Hartmann (Hg.), Regionen in der frühen Neuzeit. Reichskreise im deutschen Raum, Provinzen in Frankreich, Regionen unter polnischer Oberhoheit. Ein Vergleich ihrer Strukturen, Funktionen und ihrer Bedeutung (Zeitschrift für historische Forschung, Beiheft 17), Berlin 1994; Winfried DotZauer, Die deutschen Reichskreise (13831806). Geschichte und Aktenedition, Stuttgart 1998. - Zum Obersächsischen Reichskreis vor allem: Karlheinz BlaschKe, Der Obersächsische Reichskreis, in: Peter Claus Hartmann (Hg.), Regionen in der frühen Neuzeit, S. 127-144; Thomas NickLas, Macht oder Recht. Frühneuzeitliche Politik im obersächsischen Reichskreis, Stuttgart 2002.

2 Der sogenannte Holländische Krieg, auch Niederländisch-Französischer Krieg 1672 bis 1678. In der älteren Literatur auch als „Zweiter Raubkrieg Ludwigs XIV.“ bezeichnet, vgl. John A. LynN, The Wars of Louis XIV 1667-1714, London/New York 1999.

3 Der Kurfürst von Sachsen fungierte stets als Kreisoberster, der Kurfürst von Brandenburg war Nachgeordneter bzw. Stellvertreter, vgl. NickLas, Macht oder Recht (wie Anm. 1), S. 383. 
nituren ${ }^{4}$ hatte Kursachsen nach dem Dreißigjährigen Krieg zusätzlich geschwächt; das üppige Hofleben unter Kurfürst Johann Georg II. hatte diesen Herrscher in besondere finanzielle Abhängigkeit von seinen Landständen gebracht, die aufgrund der höfischen Festkultur Wünschen des Herrschers nach einer Aufstockung der Truppen und Militärbehörden wenig geneigt waren.

Die Einrichtung eigener stehender Heere war in der zweiten Hälfte des 17. Jahrhunderts, zumal nach den Erfahrungen mit Söldnerheeren im Dreißigjährigen Krieg, unter den Reichsständen zu einem wichtigen Politikum geworden. Im vermeintlich absolutistischen Zeitalter des Barock mit seinen zunehmenden Effizienzbestrebungen war das Militär ein geradezu ideales Mittel, einerseits den werdenden Staat machtpolitisch bis hin zum militärischen Zwang zu unterfüttern, andererseits im Zeitalter der zunehmenden Konkurrenz der Höfe, Leistungsfähigkeit bis hin zur Einschüchterung oder gar Abschreckung zu bewirken.

Die vorliegende Schrift stellt eine Mischung aus Arbeitsentwurf und Denkschrift dar, in der ein gemeinsames (militärisches) Vorgehen des gesamten wettinischen Hauses sowie die damit zusammenhängende Truppenaushebung und Finanzierung angedacht werden. In deutlicher Benennung auch der Hinderungsgründe solcher Allianzprojekte gibt die Schrift nicht zuletzt tiefe Einblicke in grundsätzliche Probleme barocker Territorien des frühneuzeitlichen Reiches. Mit hoher Wahrscheinlichkeit hat Seckendorff die Schrift auf Initiative des Prinzen und (ab 1674/75) Fürsten von Sachsen-Gotha-Altenburg, Herzog Friedrich, zu Papier gebracht. Herzog Friedrich betrieb ein militärisches Allianzprojekt aller wettinischen Linien zunächst im Rahmen des Obersächsischen Reichskreises, um nach dem Scheitern dieses Projektes den zügigen Aufbau eines stehenden Heeres in Sachsen-Gotha-Altenburg voranzubringen. In dem Arbeitsentwurf/der Denkschrift geht es in erster Linie um eine mögliche gemeinsame Kraftanstrengung aller wettinischen Linien (Albertiner und Ernestiner), um die ökonomischen und militärischen Ressourcen zusammen besser nutzen und damit den Territorialnachbarn im Reich in zunehmender Konkurrenz besser begegnen zu können (siehe 23. Konföderation des gesamten Hauses zur Reputation). Das Kernproblem (siehe 15.) liegt dabei in der Erkenntnis, dass beide Stränge - hier ein glanzvolles (sprich: teures) Hofleben, da eine effektive Landesdefension - gleichzeitig als nicht möglich erscheinen.

\section{Der Verfasser}

Der Autor der „Unmaßgeblichen Gedanken“, Veit Ludwig von Seckendorff (16261692), und sein nicht unbeachtliches publizistisches Werk und Wirken sind der Forschung seit längstem bekannt, ${ }^{5}$ wohl vor allem der Tatsache wegen, dass Seckendorff der Verfasser des wohl wichtigsten Fürstenspiegels im 17. Jahrhundert, des „Teutschen

4 Vgl. Martina Schattkowsky/Manfred Wilde (Hg.), Sachsen und seine Sekundogenituren. Die Nebenlinien Weißenfels, Merseburg und Zeitz (1657-1746) (Schriften zur sächsischen Geschichte und Volkskunde 33), Leipzig 2010.

5 Kurzbiografie und Werkkatalog sowie wichtigste Literatur: Detlef Döring, Veit Ludwig von Seckendorff, in: Neue Deutsche Biographie 24 (2010), S. 117 f., Onlinefassung: http://www.deutsche-biographie.de; neuere Biografie: Solveig STrauch, Veit Ludwig von Seckendorff (1626-1692). Reformationsgeschichtsschreibung - Reformation des Lebens - Selbstbestimmung zwischen lutherischer Orthodoxie, Pietismus und Frühaufklärung (Historia profana et ecclesiastica 11), Münster 2005. 
Fürstenstates“ (1656) ist. ${ }^{6}$ Mit diesem, seinem großen Erstlingswerk, besaß Seckendorff eine praktisch-politische Rezeption, die bis ins 18. Jahrhundert hinein anhalten sollte. ${ }^{7}$

Es sollte jedoch nicht verkannt werden, dass Seckendorff zeitlebens, auch in seinen eher staatsrechtlich-politischen Werken und seinem Denken, eine starke christlichtheologische Fundierung besaß, die nach dem Tod seines Dienstherrn, Herzog Moritz von Sachsen-Zeitz (1657-1681), immer unverhüllter zutage trat. ${ }^{8}$

Schon Seckendorffs Bestallungsverhältnis für Herzog Moritz war von der Grundvoraussetzung her nicht ganz spannungsfrei, da hier Verfassungsfragen und das auch juristische Verständnis von Kaiser und Reich berührt wurden: Bey denen teutschen weltlichen fürstenthümern und landen ist dieser merckliche unterschied wobl in acht zu nebmen, daß bey vielen, wenn der landesherr etliche söbne und erben verläst, dennoch keine theilung vorgehet, sondern die regierung der länder in gesammt behalten wird, und allezeit dem Erstgebohrenen allein zukommet [...] alsdenn insonderbeit bey den Churfürstentbümern, und denen darzu eigentlich gebörigen landen, durch die reichssatzungen es also verordnet [...]. ${ }^{9} \mathrm{Da}$ Seckendorff staatspolitisch auf dem Boden des Reiches und seiner althergebrachten, internen Strukturen stand, ${ }^{10}$ und das hieß bei Kurfürstentümern nun einmal Unteilbarkeit, ${ }^{11}$ wird er mit dem „Freundbrüderlichen

6 Der „Fürstenstaat“ ist dabei als eine Art praktisches Regierungshandbuch zu betrachten. Zur Wirksamkeit dieses Werkes auf die Regierungspraxis und die Bestallung geeigneter Fürstendiener bzw. Räte vgl. auch Christian Heinker, Die Bürde des Amtes - die Würde des Titels. Der kursächsische Geheime Rat im 17. Jahrhundert (Schriften zur sächsischen Geschichte und Volkskunde 48), Leipzig 2015, S. 132-137. Der „Fürstenstaat“ ist trotz einiger Widersprüchlichkeiten als wichtigster Fürstenspiegel zu betrachten, auch wenn Seckendorff in den acht Jahre später hinzugefügten „Additiones" manches relativiert.

7 Der „Fürstenstaat“ erlebte bis 1737 zehn Auflagen und wurde an Universitäten gelehrt.

8 Deutlich im „Christenstaat“, vgl. Detlef DöRInG, Untersuchungen zur Entstehung des "Christenstaates“ von Veit Ludwig von Seckendorff, in: Erich Donnert (Hg.), Europa in der Frühen Neuzeit. Festschrift für Günter Mühlpfordt, Bd. 1: Vormoderne, Weimar/Köln/Wien 1997, S. 477-500.

9 Veit Ludwig von Seckendorff, Teutscher Fürstenstat, Samt des Autors Zugabe sonderbarer und wichtiger Materien, Verbessert, mit Anmerkungen, Summarien und Register versehen von Andreas Simson von Biechling, Additiones, Jena 1737 (Nachdruck Aalen 1972), 2. Teil, Kap. 3, \1, S. 51.

10 Vgl. Gerhard Menk, Der deutsche Territorialstaat in Veit Ludwig von Seckendorffs Werk und Wirken, in: Heide Wunder (Hg.), Dynastie und Herrschaftssicherung in der Frühen Neuzeit. Geschlechter und Geschlecht (Zeitschrift für historische Forschung, Beihefte 28), Berlin 2002, S. 55-92. Hier vor allem zum Einfluss Seckendorffs in kleineren Territorien des Reiches, welches gleichwohl in seiner Struktur für Seckendorff den Rahmen der Politik darstellt.

11 Im Gegensatz zu DetLef DöRING, Die Höfe der Sekundogenituren in ihrer Stellung zu den geistesgeschichtlichen Entwicklungen um 1700, in: Schattkowsky/Wilde, Sachsen und seine Sekundogenituren (wie Anm. 4), S. 207-228, hier S. 209. - Zum eigentlichen Zerwürfnis mit der Kurlinie kam es erst 1681 infolge der Mitwirkung Seckendorffs am Testament von Herzog Moritz. - Die Primogenitur und Unteilbarkeit bei Kurfürstentümern war eigentlich eine conditio sine qua non im frühneuzeitlichen Reich und verdeutlicht nur einmal mehr die staatsrechtliche Fallhöhe des „Freundbrüderlichen Hauptvergleiches“. Als 1692 die neunte Kur an Braunschweig-Lüneburg (von da an auch Kurhannover) verliehen wurde, war mit der 1682 proklamierten Primogenitur nach jahrelangen Bemühungen Herzog Ernst Augusts die wichtigste Voraussetzung für 
Hauptvergleich“von 1657, in dessen Folge die herzoglichen Brüder des sächsischen Kurfürsten vom Kaiser sogar separat belehnt wurden, ${ }^{12}$ in durchaus gemischten Gefühlen Ende 1664 in den Dienst von Herzog Moritz von Sachsen-Zeitz getreten sein. Hier konnte Seckendorff als Kanzler, vor allem aber als Konsistorialpräsident und Prinzenerzieher wirken und so dem allmählichen Wandel vom Staatsrechtler, Juristen und Verwaltungspraktiker hin zum Theologen und Pädagogen noch mehr Gewicht verleihen. Von dieser Warte aus betrachtet, passten die letztlich irenischen Tendenzen Seckendorffs als Fürstendiener mit ausgeprägt christlichem Ethos nicht wirklich in eine Zeit, die immer stärker von Bellizität, und diese als gleichsam natürliche Fortsetzung der Politik mit anderen Mitteln, sowie von Konkurrenz der Dynastien und Höfe untereinander geprägt war. Dennoch erfasste Seckendorff den grundlegenden Widerspruch der kursächsischen Politik unter Kurfürst Johann Georg II. natürlich zielsicher und benannte ihn in zum Teil deutlichen Formulierungen. Als Kanzler von SachsenZeitz befand sich Seckendorff im Spannungsfeld zwischen den Ansprüchen der Sekundogenituren auf voll ausgebildete Landeshoheit; eine Forderung, die die Kurlinie stets bestritt und bei zahllosen zeremoniellen Gegebenheiten auch visualisierte. ${ }^{13}$

Seit 1669 fungierte Seckendorff auch als kursächsischer Geheimrat von Haus aus. ${ }^{14}$ Das ersparte es ihm, für Ratsverrichtungen jedes Mal nach Dresden reisen zu müssen. Von ihm wurden hingegen Gutachten und Stellungnahmen zu aktuellen politischen Vorgängen erwartet. Die Fundstelle des Entwurfes im Familienarchiv der Friesen auf Rötha lässt den Umgang Seckendorffs mit den Brüdern von Friesen (Heinrich der Jüngere und Carl) erahnen, ${ }^{15}$ zumal die Nähe von Seckendorffs Stammgut Meuselwitz einen wohl häufigeren Kontakt ermöglichte.

\section{Entstebungsumstände der Schrift}

Für Seckendorff gestaltete sich das Jahr 1672 als besonders arbeitsintensiv. So befand er sich gleichzeitig in mehreren Bestallungsverhältnissen und hatte als kursächsischer Geheimrat (von Haus aus) und als Kanzler von Sachsen-Zeitz die Verhandlungen über das altenburgische Erbe zu führen, ${ }^{16}$ das letztlich der Linie Sachsen-Gotha zufiel.

die Rangerhöhung erfüllt, vgl. Georg Schnath, Geschichte Hannovers im Zeitalter der neunten Kur und der englischen Sukzession 1674-1714 (Veröffentlichungen der Historischen Kommission zu Hannover 18), Hildesheim 1938.

12 Zum „Freundbrüderlichen Hauptvergleich“ und den daraus entstehenden Sekundogenituren vgl. auch Jochen VÖTSCH, Staatsbildung in Mitteldeutschland? Entstehung und Entwicklung der kursächsisch-albertinischen Nebenlinien, in: Schattkowsky/ Wilde, Sachsen und seine Sekundogenituren (wie Anm. 4), S. 59-72.

13 Ein Sammelband zu den Sekundogenituren heißt nicht ohne Grund „Fürsten ohne Land“, vgl. Vinzenz Czech (Hg.), Fürsten ohne Land. Höfische Pracht in den sächsischen Sekundogenituren Weißenfels, Merseburg und Zeitz (Schriften zur Residenzkultur 5), Berlin 2009. Der Sammelband stellt den im Zeitalter des Barock auch politisch interpretierten Repräsentanzaspekt und seine verschiedenen Visualisierungen in den Vordergrund und verdeutlicht so nicht zuletzt, dass es den Sekundogenituren eben an allseits akzeptierter Landeshoheit gebrach.

14 Bestallung vom 2. Januar 1669, vgl. Sächsisches Staatsarchiv - Hauptstaatsarchiv Dresden, Geheimer Rat (Geheimes Archiv) 10024, Loc. 7169/28, fol. 234.

15 Um 1670 die führenden Geheimräte in Kursachsen, vgl. Heinker, Geheimer Rat (wie Anm. 6), S. 318 f. und passim.

16 In diesem Zusammenhang ist der intensive Kontakt zu Herzog Friedrich von SachsenGotha von Bedeutung, der noch zu Lebzeiten seines Vaters Ernst ab 1674 diesem in der 
Außerdem verfasste Seckendorff für seinen gewesenen Dienstherrn, Herzog Ernst (den Frommen) von Sachsen-Gotha, einen Traktat über die Führung eines Gymnasiums. ${ }^{17}$ Weiterhin wohnte Seckendorff ab 1672 wohl ständig auf dem Gut Meuselwitz, ein Rittergutssitz, den er 1676 endgültig erwarb und dort ein Gutshaus errichten ließ. Außerdem wollte Herzog Ernst von Sachsen-Gotha, der inzwischen wohl erkannt hatte, welche Kapazität er 1664 verloren hatte, Seckendorff Ende 1672 zurückgewinnen und bot ihm die neu geschaffene Stellung eines Geheimratspräsidenten von Sachsen-Gotha-Altenburg an, eine Position, die Seckendorff jedoch ablehnte. ${ }^{18}$

Zum Zeitpunkt der Abfassung der Schrift (13. Juli 1672) wurde in Leipzig ein Obersächsischer Kreistag abgehalten, an dem Seckendorff sehr wahrscheinlich nicht teilgenommen, ${ }^{19}$ den Ausgang dieser Versammlung mit Sicherheit jedoch aus der Nähe beobachtet hat, da das Kernproblem des Entwurfes, eine möglichst schlagkräftige Aushebung von Kreistruppen zur Abwehr der französischen Armee vom Reichsterritorium, dort diskutiert wurde. Zur Beratung stand eine gesamtwettinische Allianz unter Einschluss der ernestinischen Linien in Thüringen, die die Schlagkraft der Obersächsischen Kreistruppen erhöhen, aber auch den Kreisvorsitz des Kurfürsten von Sachsen untermauern sollte. So war eine Versammlung der Haupt- und Nebenlinien zur „Wehrverfassung“ 1672 geplant, zu der Seckendorff den Entwurf lieferte. ${ }^{20}$ Hier konnte Seckendorff in besonderer Weise die Arbeitsmaterie überschauen und gewesene und aktuelle Bestallungsverhältnisse und daraus resultierende Kontakte und Netzwerke beinahe idealtypisch nutzen. Als ehemaliger Kanzler Herzog Ernsts von Sachsen-Gotha, engem Korrespondenzpartner von dessen Nachfolger, Herzog Friedrich, aktuellem kursächsischem Geheimrat von Haus aus und Kanzler von SachsenZeitz verfügte Seckendorff über fast alle Positionen und Kontakte, die ein großflächiger Überblick über ein gesamtwettinisches Projekt zu erfordern schien. Selten waren im 17. Jahrhundert die Mehrfachloyalitäten ausgeprägter als in diesem exponierten Beispiel. Als erfahrenem politischen Beobachter ${ }^{21}$ muss Seckendorff klar gewesen sein, dass nur eine gesamtwettinische Anstrengung den dauerhaften Vorrang Kursachsens im Obersächsischen Reichskreis gegenüber Kurbrandenburg würde behaupten können. Für Kursachsen waren die Voraussetzungen, dies auch ins Werk setzen zu können, unter dem Kurfürsten Johann Georg II. indessen denkbar schlecht. So blühte

Regierung nachfolgte. Der Austausch ist über das Tagebuch Herzog Friedrichs und dem Briefwechsel zwischen beiden Korrespondenzpartnern besonders gut belegt, vgl. Friedrich I. von Sachsen-Gotha und Altenburg. Die Tagebücher 1667-1686 (Veröffentlichungen aus thüringischen Staatsarchiven 4), bearb. von Roswitha Jacobsen, unter Mitarbeit von Juliane Brandsch, 3 Bde., Weimar 1998-2003. - Etwa drei Viertel des Altenburger Territoriums fielen an Sachsen-Gotha.

17 Vgl. Roswitha Jacobsen, Die Brüder Seckendorff und ihre Beziehungen zu SachsenGotha, in: Dies./Hans-Jörg Ruge (Hg.), Ernst der Fromme (1601-1675). Staatsmann und Reformer, Wissenschaftliche Beiträge und Katalog zur Ausstellung, Bucha bei Jena 2002, S. 95-120, hier S. 110.

18 Vgl. ebd., S. 109.

19 Sachsen-Merseburg und Sachsen-Zeitz stellten keine Abgeordneten, vgl. den Text des Entwurfes unter Punkt 3.

20 Kurzer Anriss des Themas in: Frank Boblenz, Albertiner und Ernestiner, in: Hans Hoffmeister/Volker Wahl (Hg.), Die Wettiner in Thüringen. Geschichte und Kultur in Deutschlands Mitte (Schriften des Thüringischen Hauptstaatsarchivs Weimar 2), Weimar/Arnstadt 1999, S. 95-100.

21 Vgl. Rudolf Schlögl, Politik beobachten. Öffentlichkeit und Medien in der Frühen Neuzeit, in: Zeitschrift für historische Forschung 35 (2008), S. 581-616. 
unter diesem Herrscher eine üppige Festkultur,22 die im Zeitalter des Barock zwar auch politisch zu interpretieren war, ${ }^{23}$ jedoch Finanzkalamitäten verursachte, die eine stabile Wehrverfassung mit Kreistruppen, ein stehendes Heer oder ein gesamtwettinisches Militärprojekt von Beginn an unter einem ungünstigen Stern erscheinen ließen. Das erforderliche Maßhalten zum Erhalt ausgeglichener oder zur Gesundung der Staatsfinanzen lässt sich in Seckendorffs Denken leicht nachweisen.

So bildet der hier vorliegende Entwurf Seckendorffs eine letztlich nicht realisierte und nicht realisierbare Möglichkeit, innerhalb des Reichsrahmens militärisch aktiv zu werden und Stärke zu zeigen. Da dies in Kursachsen schon bald auf Ebene des Territoriums mit der Schaffung des ersten stehenden Heeres möglich wurde, setzten sich letztlich die zentrifugalen Kräfte durch, die durch die militärisch unterfütterte Machtpolitik der Territorien den Reichsrahmen als überlebte und entbehrliche Größe erscheinen ließen, sobald es um die praktische und pragmatische Politik ging.

\section{Nachwirkung}

Der Kontakt bzw. briefliche Austausch zwischen Seckendorff und Herzog Friedrich von Sachsen-Gotha-Altenburg blieb über 1672 hinaus sehr intensiv. ${ }^{24}$ Herzog Friedrich gebot ab 1674/75 als Nachfolger seines Vaters Ernsts des Frommen mit der Altenburger Erbschaft über zwei Drittel des wettinischen Territoriums in Thüringen und stand damit der bedeutendsten ernestinischen Linie vor. ${ }^{25} \mathrm{Als}$ er 1691 starb, besaß sein Land ein für seine Verhältnisse ziemlich überdimensioniertes Heer von 10000 Mann, für das er fast alle finanziellen Mittel einsetzen musste. Dies fiel ihm sicherlich leichter, da der „hausväterliche“ Hof seines Vaters als adelsfern und bescheiden einzustufen war. ${ }^{26}$ Dies hatte Seckendorff im „Fürstenstaat“ als Tugend propagiert, die Fürsten mittlerer Territorien gut zu Gesicht stehe.

So muss wohl Herzog Friedrich als geistiger Ahnherr und treibende Kraft der gesamtwettinischen Allianz betrachtet werden, wenn er die finanziellen Ressourcen seines im Reichsmaßstab dann doch recht kleinen Territoriums bis an die Grenze des Erträglichen strapazierte für ein Heer, das in seiner Personalstärke dem ersten sächsischen Heer von 1682,27 immerhin eines Kurfürstentums, nur wenig nachstand.

22 Deutlich: Uta Deppe, Die Festkultur am Dresdner Hofe Johann Georgs II. von Sachsen (1660-1679) (Bau + Kunst. Schleswig-Holsteinische Schriften zur Kunstgeschichte 13), Kiel 2006.

23 Vgl. Christian Horn, Der aufgeführte Staat: zur Theatralität höfischer Repräsentation unter Kurfürst Johann Georg II. von Sachsen (Theatralität 8), Tübingen/Basel 2004.

24 Vgl. Jacobsen, Friedrich I. von Sachsen-Gotha und Altenburg (wie Anm. 16). Besonders in Bd. 1 (1667-1677) sind die brieflichen oder persönlichen Kontakte für 1672 als beinahe täglich zu bezeichnen; am 8. Juli 1672 erhielt Herzog Friedrich ein Schreiben von Seckendorff wegen Zusammensetzung des Hauses, am 13. Juli 1672 bekam Herzog Friedrich zwei bacqvet vom Kreistag, zwei Tage später wiederum eines, am 16. Juli besucht der Dienstherr Seckendorffs, Herzog Moritz, Friedrich, vgl. ebd., Bd. 1, S. 240 f.

25 So führte er als erster Ernestiner 1685 die Primogenitur ein, ein Beispiel, dem bis ins 18. Jahrhundert die anderen Territorien nach und nach folgten.

26 Vgl. Andreas Klinger, Der Gothaer Fürstenstaat. Herrschaft, Konfession und Dynastie unter Herzog Ernst dem Frommen (Historische Studien 469), Husum 2002, S. $144 \mathrm{f}$.

27 Vgl. Walther Thenius, Die Anfänge des stehenden Heerwesens in Kursachsen unter Johann Georg III. und Johann Georg IV. (Leipziger Historische Abhandlungen 31), Leipzig 1912. 
Schon zuvor, 1681, war mit der sogenannten Reichsdefensionalordnung die endgültige Form gefunden worden, mit der die Heeresverfassung und der Beitrag der Territorien zumindest formal bis zum Ende des Alten Reiches geregelt war. ${ }^{28}$ Diese hatte schon bald im erneuten Reichskrieg gegen Frankreich ihre Bewährungsprobe zu bestehen. Somit war auch ein Grund für die Abhaltung von Kreistagen weggefallen, die für den Obersächsischen Reichskreis tatsächlich seit 1683 nicht mehr stattfanden.

Im Streit um die Führung der Kreistruppen gelang Kursachsen 1674 ein letzter Prestigeerfolg, als der Dienstherr Seckendorffs, Herzog Moritz von Sachsen-Zeitz, die Kreistruppen ins Feld führen durfte, allerdings ohne Beteiligung Kurbrandenburgs, das sogar versuchte, das Kreiskontingent seinem Kommando einzuverleiben. ${ }^{29}$ So wurde durch die Mächtekonkurrenz, hier die besondere zweier Kurfürstentümer in einem Reichskreis, letztlich der Zerfall der Kreisverfassung herbeigeführt.

Seckendorff hingegen zog sich 1681 endgültig aus der Politik mit ihren Ratsverrichtungen zurück und widmete sich seiner eigentlichen Berufung, der Theologie. Nach dem Tod von Herzog Moritz wurde deutlich, dass er maßgeblich Mitwirkender an dessen Testament war, das die Ansprüche der Kurlinie zurückwies. So fiel er bei dem neuen sächsischen Kurfürsten, Johann Georg III., in Ungnade, was das Erlöschen seiner Bestallungsverhältnisse mit sich brachte. ${ }^{30}$

Seckendorff führte bald u. a. einen erbitterten Streit unter Gelehrten ${ }^{31}$ mit seinem Gegenspieler, Samuel Pufendorf (1632-1694),32 der mit seinem Hauptwerk De jure naturae et gentium, das 1672 erschienen war, separate Bereiche für (Staats-)Philosophie und Theologie reklamierte, die Reichsverfassung für weitgehend irregulär hielt und auf Basis des Naturrechts systematisierend auf Staatsbildung und die frühneuzeitlichen Rechtsverhältnisse insgesamt prägend wirken konnte.

Kurfürst Johann Georg II. von Sachsen hatte sich für eine glänzende Hofhaltung entschieden, Herzog Friedrich von Sachsen-Gotha-Altenburg für ein - gemessen an der Größe seines Territoriums - überambitioniertes Heer. Beide Möglichkeiten führten $\mathrm{zu}$ maroden Finanzen und einem Schuldenberg. Mit dem Kameralismus alter Schule, ${ }^{33}$ als dessen Vertreter Seckendorff auch galt, und das hieß Maßhalten, Sparen und Kostenvermeidung, war den Necessitäten der barocken Staatenwelt nicht mehr beizukommen.

28 Vgl. Gerhard Papke, Von der Miliz zum Stehenden Heer. Wehrwesen im Absolutismus, in: Deutsche Militärgeschichte in sechs Bänden. 1648-1939, hrsg. vom Militärgeschichtlichen Forschungsamt, Bd. 1, München 1983.

29 Vgl. Nicklas, Macht oder Recht (wie Anm. 1), S. 284.

30 Seckendorff musste gar zeitweise um seine Ansprüche und Pensionen für seine Tätigkeit für Sachsen-Zeitz fürchten, vgl. Jacobsen, Die Brüder Seckendorff (wie Anm. 17), S. 113.

31 Ein bemerkenswerter Streit, da beide keinen direkten Briefwechsel unterhielten und der Konflikt mithin über Dritte ausgefochten wurde, vgl. STRAuch, Veit Ludwig von Seckendorff (wie Anm. 5), S. 154-162. Seckendorff argumentiert weitgehend christlichtheologisch, aber auch politisch, indem der Kampf der Christenheit gegen den türkischen Feind propagiert wurde, Pufendorf hingegen eher juristisch-sachrational und säkularistisch.

32 Vgl. Dieter Hüning (Hg.), Naturrecht und Staatstheorie bei Samuel Pufendorf (Staatsverständnisse 23), Baden-Baden 2009.

33 Vgl. JutTa BRÜCKNer, Staatswissenschaften, Kameralismus und Naturrecht. Ein Beitrag zur Geschichte der politischen Wissenschaft im Deutschland des späten 17. und frühen 18. Jahrhunderts (Münchner Studien zur Politik 27), München 1977. 


\section{Edition}

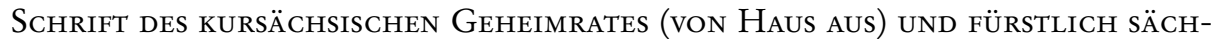
Sisch-Zeitzischen Kanzlers Veit Ludwig von SeckendorfF

Ohne Ort (vermutlich Zeitz oder Rötha), 13. Juli 1672.

Quelle: Sächsisches Staatsarchiv - Staatsarchiv Leipzig, 20532 Rittergut Rötha mit Trachenau, Nr. 1547; Unmaßgebliche Gedanken über die vorhabende Zusammensetzung des Chur- und Fürstl. Hauses Sachßen, und was davon dependiret, fol. 145r-165r, ungedruckt (Arbeitsentwurf bzw. Denkschrift Seckendorffs zu einem Allianzprojekt des gesamten wettinischen Hauses).

Die Wiedergabe des Entwurfes erfolgt wort- und buchstabengetreu. Geringeren Eingriffen in Zeichensetzung und Rechtschreibung steht eine Vereinheitlichung der Großund Kleinschreibung gegenüber. Hervorhebungen des Verfassers im Text stehen zwischen ${ }^{*} \ldots *$.

1.

[fol. 145r] Der Zweck bestehet sonder zweifel in Erhaltung der samtlichen Lande und Leute, daß dieselbe vor feindlichem Anfall bewabret, und be y Ibrer verfaßung in Christ = und weltlichen Sachen beschützet werden: Nechst diesem Hauptabsehen wird ungewebrt seÿn, mittelst solcher Zusammensetzung, auf die Verbeßerung des Estats und Behauptung eines und andern rechtmäßigen Desider $\ddot{y}$, so das gesamte Hauß und deßen glieder haben mögten, darunter die Jülichische Successions=Sache das vornebmste së̈n kann, ein abseben zu haben.

\section{2.}

Zu diesem Zweck hetten nun zu concurriren zuvörderst Churfürstl. durchl. zu Sachßen, nach dero bekanten und von dero Herrn Brüdern und Vettern unschwehr agnoscirten praerogativ, alßo daß derselben sowobl [fol. 145v] von dero Herren Brïdern dasjenige, was der Hauptvertrag vermag, und von den Herren Vettern der Fürstl. Linien das Directorium obne difficultet gegönnet werden wird; So ist auch Gott lob von alten und neuen praetensionen unter denen Chur= und Fürsten des Haußes selbsten nichts wichtiges mehr vorhanden, darüber sich solche Zusammensetzung splittern, oder impracticirlich fallen könnte. Ist alßo allein zuseben, wie die media zu solchen heilsamen Zweck zuerfinden, und auf was nützliche art und weiße solches alles zuverfaßen.

\section{3.}

Davon zu tractiren, wird der gelegenheit ietzigen Crë̈ßtages für sebr dienlich gebalten, und obwobl die beÿden Herren Hertzogen zu Sachßen Merßeburg und Naumburg keine Gesandte allda haben, so könten doch dieselben obne Solennitet einen oder zwe ÿ dero vertrauten Räthe hierin schicken, und sowobl mit den Churfürstlichen, alß des [fol. 146r] fürstl: Haußes Gesandschaften unterschiedlich conferiren, dabë̈ es denn, vielerleÿ formaliteten zu vermeiden, keines allgemeinen Consessus bedörfen wird, sondern à part durch Conferenz und Discurse biß zu einem Project gehandelt werden könnte; Chur= und Fürstl. Herrschaften aber würden in der nähe seÿn, oder die Posten zu Erlangung schleuniger Resolution füglich bestellen lassen, auch Räthe bë̈ sich haben, mit denen Sie alles überlegen und schließen könten, und da einer oder anderer Theil, alß mann sich doch nicht versehen kan, anfangs birzu nicht zu disponiren, so könnte doch von den meisten biß zum project fortgefabren werden. 
4.

Das vornehmste Stük, ia das wesen und die form der Zusammensetzung bestehet in Vereinigung der Rathschläge, daß das gantze [fol. 146v] Hauß einerlë̈ Zweck habe, in Krieg und Friedenszeiten sich miteinander unzertrennet zu conserviren, innmaßen Sie darzu die Blutsverwandtschaft, und zu unterschiedlichen mablen verneuerte Schwägerschaft, gesamte Belebnung, und Succession, auch Situation und Vermengung der Lande, nichts weniger einerleÿ Religion und Landrecht gnugsam animiret auch die böchste Noth antreibet, weil fast kein Nachtbar vorhanden, mit dem mann in dergleichen Vinculis sich verbunden findet, und dem an der Conservation dieser Lande ein mebrers gelegen, jenes wird vielmehr durch den Unterschied der Religion, und allerleÿ mit denselben habende Differentien und gantz wiedrige interessen dieses Chur= und Fürstl. Hauß destomehr veranlasset, ibre Consilia und Kräfte zusammen zubalten.

5.

Soll nun eine Conjunctio Confitiori [fol. 147r] mit Bestand gestiftet werden, so muß conjunctio animorum vorber fest stehen, und alßo auß dem wege geräumet werden, was die Gemüther der Chur= und Fürsten gegen einander zu Mißtrauen, Wiederwillen, Zank und Streit treiben und bewegen kan, oder ist zum wenigsten ein bequebmes mittel zu stabiliren, wodurch die entstehende Irrungen alßo fort gütlich und nach Billigkeit obne Weiterung zuerörtern.

6.

Zu solchem Zweck dienet die Erneuerung der Erbverbrüderung, und Einrichtung gewißer Mediation und außträglicher Erörterung aller im Hauße fürfallender Irrungen worzu, was nicht eben itzo sobald, wie es etwas Zeit und Uberlegung erfordert, doch mit dem ehisten zuschreiten bedacht und vorbehalten werden könnte.

7.

Zu Faßung gemeiner $=$ und $z u=[$ fol. 147v] sammengesetzter Rathschläge geböret auch die Bestellung solcher Räthe und Diener, welche zu diesem Scopo, und Behauptung deßen, was verglichen wirdt, mit Hindansetzung aller andern Respecten, wobl intentioniret, auch verpflichtet seÿn müsten, damit einer dem andern trauen dörfte, und nicht zubefabren stebe, daß mann wieder die Principia disputire, oder einer dieser, der andere jener Religion und faction së̈, oder von außwertigen Potentaten seine Fortun und Aufnebmen suche.

8.

Es geböret auch darzu eine Correspondenz und einerle y Absehen der samtlichen Landschaften die weil zumahl die von der Ritterschaft in den Chur= und Fürstenthümern durch Geschlecht, Heÿrathen mit Erbschaften und Nachbarschaft einander vielfältig verbunden, so dienet zu Erhal= [fol. 148r] tung zusammenstimmender Rathschläge nicht wenig, wenn die vornehmsten Personen auß dero Mittel einerle $\ddot{y}$ Zweck, Information und Principia haben, und alßo auf den Landtägen, die gute Intentiones allentbalben aus einerleÿ Motiven und Principÿs fördern können, auch eine mügliche Gleichbeit der Bürden und Lasten eingefübret wirdt, dann wo sonst der einen Land allzu boch, des andern zu wenig angegriffen wird, da entstehet in Friedenszeiten Unwillen und Aemulation, im Krieg aber andere und höhere Incommoda, welche die Noth mit sich bringet, aus welchen allem folget, daß zwischen Herrschaften und Unterthanen, und zwischen den Unterthanen selbst gutes Vernebmen nach aller Mügligkeit gestiftet und erbalten werden müßte, wann mann einen so beilsamen Zweck daurbaft und mit Hofnung [fol. 148v] guten Successus erbalten will. Diejenige aber, welche die Herrschaften und Unterthanen zusammen betzen, und über all berkommen, Pacta und Reversalien zuschreiten persuadiren wollen, (müßen) vor solche Leute gehalten wer- 
den, * die nur ibren eigenen Nutzen, und der Herrschaft und Landes wabren und beständigen Nutzen nicht für Augen haben, denn nimmermebr obne gute Harmonie zwischen Herren und Landen, und da jeder be y dem Seinigen ungekränkt bleiben kan, einig Exempel zu allegiren, daß es wobl und glücklich abgegangen, hingegen alle Erfabrung bezeüge, was große und nützliche Dinge zumahl in diesen Landen, durch Zusammentretung der hohen Obrigkeit und der getreuen Landschaft wieder iedesmablige Feinde in den allergefährlichsten Zeiten außgerichtet worden. *

\section{9.}

Nach diesen Praesuppositis ist dann [fol. 149r] von den außerlichen Mitteln zureden, und bestehet darinn die gröste Difficultet, als deren Ursachen zuerforschen, und Remedia dargegen zufinden, sehr gehäßig und gefährlich ist, gleichwohl nachdem es solche Wege erreichet, daß, wo mann anderst etwas nützliches be ÿ anscheinender außerster Noth zu Rettung und Hülfe der Herrschaften und Unterthanen thun, und nicht gar an allen gedej̈en verzweifeln will, die Krankheit erkennet und genennet së̈n muß: So wird im Nachdenken und weiser Uberlegung Niemand verdacht werden können, der hierunter obne Verliebrung des denen hoben Obrigkeiten schuldigen Respects, und allein aus unterthänigster Liebe und Vorsorge für die gemeine Woblfabrt, Frej̈beit nimmet, die Nothdurft zueröfnen, und wie in Heilung eines kranken [fol. 149v] Leibes alles vergeblich ist, wann der Artzt nicht die Ursachen des Schadens oder der Krankbeit ergründet, alßo ist in dieser politischen Schwachbeit gantz unentbebrlich zuerforschen, woher doch der offenbabre und von männiglich hochgeklagte Mangel der Mittel zu gemeiner Landes Verfassung und Defension entstanden së̈, und noch entstebe, denn daraus wird leicht zuschließen seÿn, ob und wie demselben abzubelfen.

10.

Mann muß nun anfangs bekennen, und kan von denen, welche die Geschichte der Vorfabren gelesen und zubertzen genommen, nicht gelengnet werden, daß zwar in diesen Landen ie und allwege über den Geldmangel geklaget, solche Klage aber ie länger ie beftiger worden, und gleichwobl, sonderlich [fol. 150r] in vorigen Seculo, und dem Anfang des ietzigen, niemahls so viel an allerhand Stenern und Anlagen, alß itzo von denen Unterthanen begebret und erlanget worden, dabero viel Leute bë̈ Höfen sich finden, welche dergleichen Klagen nur für eine angeborene Gewobnheit der Landstände und Unterthanen halten, und großen Potentaten be j̈bringen wollen, daß sie sich daran nicht kebren mögten. Nechst diesem werden die Historien und Acta geben, wie das wenige, so mann gleichwohl nicht obne große Difficultet anfangs an Steuern erboben, von Zeiten zu Zeiten erböbet, und nun auf eine solche Maße hinauf getrieben worden, daß mann vermeinet, es seÿ solche ferner zutragen, geschweige zuergrößern unmüglich, und nichts [fol. 150v] destoweniger muß mann auch gesteben, daß zu jenen Zeiten, da sowobl die Fürstl. Cammerrenten ein wenigers alß ietzo eingetragen, und die Mittel des Landes, alß Getreide, Wolle, Leinwand, Holtzwabren, auch Ertz und Mineralien oftermabls noch woblfeiler als ietzo gewesen, dennoch große Dinge (vorgangen) gescheben, gantze Armeén aufbracht, Krieg geführet, Vestungen gebauet, Zeughäußer angerichtet, ansebliche Gebäude geführet, und kostbabre Mobilien gezenget worden, auch milde Stiftungen, und zu weilen nicht geringe Donationes gescheben, und doch wobl ein bahrer Vorrath be y Handen gewesen, an welchen allen es ietzo mangelt, und dennoch das Land ungleich höher, als jemahls, angeleget wird.

\section{1.}

Eine wabre und männiglich [fol. 151r] für Augen liegende Ursach ist in den Churfürstl. Landen die allzu große auf der Landschaft liegende Schuldenlast, die zwar größten Theils aus unterth(änig)ster Trene und Guthertzigkeit durch die bekante Ubernebmung so vieler Tonnen Goldes Cammerschulden in guten Zeiten entstanden, aber bald 
durch ein brechenden dreÿßigjährigen Krieg im Zweck dergestalt verruckt worden, daß mann in effectu solche Last bißher nicht mindern können, ja ietzo noch weit höher, alß etwann An. $1628 \mathrm{zu}$ Zeit solcher Ubernehmung, beschwebret ist, und dabero müssen nun jäbrlich auß dem Churfürstenthum Sachßen und darzu gebörigen Landen über zweÿmabl bunderttausend Gülden allein an Zinßen aufgebracht und weg gegeben werden, [fol. 151v] obngeachtet denen allermeisten Creditoren nur 3. von 100. und vielen nichts gereichet wirdt, und ist gleichwobl weder Christlich noch den Rechten gemäß, denen jenigen, die Ihre Mittel darzu hergeliehen, Capital und Zin $\beta$ zu Waßer zumachen, werden auch die Chur= und Fürstl. Barschaften und die meisten Communen und pia causa darüber den größten Schaden leiden, darumb were wobl das rechte Mittel gewesen, dem Land NB*wieder zukräften zubelfen, daß mann nach geschloßenen Friede jäbrlich nur eine Tonne Goldes effective abzulegen sich resolviret, und den Haußhalt darnach eingerichtet hette, es würde ietzo das meiste abgezablet, und anstatt deßen, so mann zu Zinßen haben muß, die Steuer zur Landesrettung anzulegen seyn, wie dann, so lang diese Last* nicht vom Land kommet, kein recht ersprießliches Mittel [fol. 152r] zu deßen Aufnebmen zu erseben.

12.

Will mann aber weiter gehen, und die erste Ursache dieser Schuldenlast, wie auch des noch beute immerwebrenden und vermebrten Unraths nicht dissimuliren, so ist, nechst denen Unkosten des verwichenen Kriegs, (umb deßentwillen die Steuerschulden unabgelegt blieben) die vornehmste: der große *Aufgang be y Hofe, welchen zwar trene Räthe und Unterthanen zu allen Zeiten kläglich angezogen, und umb deßen * Remedirung inständig gebeten, aber biß dato so gar nichts erbalten, daß Sie nunmebro davon zureden fast gantz abgeschreket worden, und gleichwobl ist kein Mittel übrig noch einige Verbeßerung zuhoffen, so lang diese Hauptquell alles Verderbens nicht gestopfet wird; und obwobl von Stüken zu Stüken, in Gegeneinanderbaltung der vorigen [fol. $152 \mathrm{v}]$ und ietzigen Zeiten gar leicht were, anzuzeigen, wie alles doppelt und mehrfältig, es seÿ Speiße, Trank, Kleidung, Anzabl und Besoldung der Diener beÿ Höfen und auf dem Lande, bë̈ denen Collegÿs und der Miliz, und alßo allenthalben, auch an allen andern Außgaben gestiegen, so ist doch unnöthig, in öffentlichen und kundbaren Sachen mit Verdruß sich aufzubalten: Alle ebrliebende Gemüther seufzen darüber, und es ist unmüglich, daß Christliche bohe Landesfürsten das Übel nicht sehen und merken sollten, nur feblet es an einer dapfern und beständigen Resolution, demselben zu begegnen, und so lang dieses nicht geschiebet, und von den Höfen auß, der in das Land gleicher Gestalt beÿ Adel und Bürgerstand eingeflochtene Luxus, Eitelkeit und Verthuligkeit, (:da die meisten unläugbar an ein einig Kleid, [fol. 153r] oder auf ein Gelag oder Kartenspiel mehr wenden, als Sie in vielen Jahren, und wohl die Zeit Ihres Lebens zu gemeinen Nutz nicht contribuiret:) nicht angegriffen und verfolget wird, so ist alle Zusammenstimmung der Rathschläge unfruchtbar, weil mann sich selbst alle Mittel abschneidet und verderbet, die mann zu Beschützung und Rettung des Vaterlandes anwenden könnte und sollte, auch die löblich *Vorfabren angewendet und Ihre Cammereinkünften darzu vornebmlich mit gebrauchet haben, wie die Rechnungen weisen werden. *

13.

Will mann auch so weit nicht gehen, sondern nur die Länder betrachten, worinn Zeit her des geschlossenen Friedens die vor= und in dem Krieg aufgewachsene Cammer= und [fol. 153v] Landesschulden bezablet, neue und kostbare Dinge hingegen geschafet und Vorrath erlanget worden, und da mann gleichwohl die Landtschaften nicht halb so boch, altz ietzo mit Steuern und andern Bewilligungen angegriffen, so ist nechst Gottes Segen einig und allein die Sparsamkeit der Höfe, und darnach eingerichtete beßere 
Haußhaltung der*Unterthanen, samt Beförderung der Commercien, darauf niemand mit Ernst ietzo denket, * die Ursach darzu gewesen, und dabero offenbabrlich, daß mann einig und allein durch solche Mittel, wiewobl alles nach geböriger Proportion und obne allzu enge, und schimpfliche Einspannung der Chur= und Fürstl. Herrschaften, zu gleichmäßigen Zwek gelangen könnte. [fol. 154r]

\section{4.}

Wiewobl nun schwebr und fast desperat ist, ein solch eingerißenes Ubel auß dem Grunde zu beben, und die jenige, so davon reden, nichts alß den höchsten Undank und Spott, wegen der großen Menge und Autoritet derjenigen, die mit gemeinen Schaden sich nebren, und der Gütigkeit der theuren Landesfürsten missbrauchen, davon zugewarten, so ist doch kein ander, alß dieses Mittel durch Menschen Witz und Fleiß zuersinnen, alß $N B * d a \beta$ der Aufgang der Höfe in allen Capiteln von Grund auß reformiret werde, es möge auch treffen und wehe thun, wen es wolle: alles andere, was mann ersinnet, lauft auf neue Beschwehrung der Unterthanen hinauß, und [fol. 154v] bestehet auf den ungegründeten, wieder Gewißen und Wabrbeit laufenden Fürbildungen, alß ob die Länder obne Unterscheid noch höher angegrifen werden könten, * und ist leider zu besorgen, so lange mann diese Meinung nicht fabren läßet, daß Gott der Herr, und die ofenbabre Noth eine solche Probe Unß in die Hände geben werde, deren kläglichen Effect wir und die Nachkommen nimmermehr verwinden werden können.

15.

Derowegen wird hierunter eine Resolution zufaßen; oder (bingegen) einzuraumen und zu gestehen seyn, daß alle gute Consilia vergeblich, und von keiner Verfaßung oder Defension zureden seÿn, wo keine Mittel darzu aufzubringen, [fol. 155r] und daß nimmermehr beÿsammen steben könne, den bißherigen Aufgang der Höfe, fast einig und allein auf der Lande Kosten fortzusetzen, und noch etliche tausend Mann Krieges Völker zu der gemeinen Landesdefension zu unterbalten. In allen dergleichen Gefabren lebret die Vernunft, daß mann zum Exempel, Geld und Gut fabren, und sein Leib und Leben salviren, lieber das Interesse als das Capital einbüßen, lieber mit einem Stük Brod, oder Haussmanns Kost vorlieb nebmen, als gar Hunger leiden, und, wie das Sprichwort saget, lieber armselig reiten, alß stoltz zufuße gehen wolle, nur allein be y denen Höfen will keine solche Lebre gelten, sondern mann will, ungeachtet Gott täglich und augenscheinlich die Mittel entzeubet, [fol. 155v] von keiner Lust und Bequemligkeit absteben, sondern, biß zum außersten Untergang, seinen Willen haben, ja noch immer mehr und mehr aufwenden, und alle Jahr die onera des Landes erböhen.

16.

Zwar ist ein Land und Fürstenthumb leidlicher, alß das andere belegt, und möchte seÿn, daß dieses oder jenes Stük Landes noch etliche Stenerpfennige aufbringen könte, es wird aber damit zum Hauptwerk gar ein weniges beÿgetragen werden können und gleich wie das Churfürstenthumb und incorporirte Lande wohl beÿ nabe drë̈ Viertel gegen * die Fürstl. Lande außmachen, so fället auch die größeste Last auf dieselbe, und müßte * alßo diese (jenes) zum Exempel drë̈ Tonnen Goldes aufbringen, da [fol. 156r] jene (diese) mit einander nur eine verschafen, derowegen bestebet die Woblfabrt des gantzen Haußes umb so viel desto augenscheinlicher auf dem Woblstand der Churfürstl. Lande, je größer und mächtiger die selbigen sind, und denen andern Hülfe und

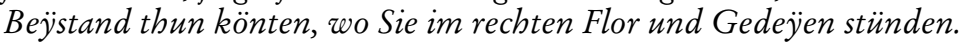

\section{7.}

Wann nun der Zustand dieser der Churfürstl. Lande nach den ietzigen Anlagen erwogen, und darbë̈ (etliche wenige Orter und kaum ein bar Stätte außgenommen) sonnenklar demonstriret werden kan, daß bey ietzigen ordinar= und extra- 
ordinar=Steuerpfennigen, Tranksteuern, Quatembern, Fleischpfennig die Unterthanen schon von allen Kräften kommen, die Häußer in den meisten Stätten zu feiten [fol. 156v] woblfeilen Kauf stehen, und kein Vorrath noch mobilien, darauß auch durch die schärfesten Executioner Geld zu lösen, vorhanden, ja welches fast unglaublich, daß in den besten Friedens Jabren bie und da die Güther auf dem Lande wieder anfangen wïst und herrenlo $\beta$ zu werden, so wird die Rechnung leicht zumachen seyn, daß mann entweder vom Land nichts mehr begebren könne, oder wenn es gleich auf dem Papier verwilliget würden, dennoch ein viel mebrers nicht ein zubringen, und hingegen die Cammer Gefälle ins Steken gebracht, und denen Herrschaften solche entzogen, consequenter diese zu noch größerer Noth und Beschwebrung veranlaßet werde.

18.

Mann laße aber seÿn, daß noch über die bißherigen alßo ge= [fol. 157r] nante Quatember, noch so viel aufzulegen, daß mann alle Monathen einen geben solle, (: obs müglich wird der leidige Eventus zeigen :) so wird es nicht viel über eine Tonne Goldes des Jabrs (mebr alß iebe) außtragen, solten nun die Crë̈ßvölker daran erhalten werden, in deren Aufbringung mann ietzo begrifen, und von den andern Verpflegungen nichts abgeben, noch zu dieser Verfaßung gebrauchet werden können, was Churfürstl. Durchl. für dero Kriegs Estat erbebet, und über zweÿ Tonnen Goldes jährlich laufet, ja wann auch zu der dritten Tonnen Goldes nach dieser Proportion gleich die Fürstl. Linie 30000 Thl. verschafte, so wird so ein weniges vor so ansehnliche Lande herauß kommen, daß mann kaum eine Anstalt zu Wartgeldern und Defensionern davon erbalten könnte, welche aber zu diesem Zwek nicht zulänglich. [fol. 157v]

19.

Mann setze, welches doch gar gering und verächtlich scheinet, es solle das gantze Chur= und Fürstl. Hauß zum allerwenigsten II 1500 geworbene Reuter und 4000 Fußknechte II beständig auf den Beinen haben, für einen Reuter 6 Thl. Monathlich, und für einen Fußknecht 3 Thl. gerechnet, erfordert es monathlich nur für die Gemeinen 21000 Thl., und für die Officiere, alß obngefehr 5 oder 6 Obristen, etlich und funfzig Ritt Meisters und Capitaine, so viel Leutenants und Fähndrig, oder Cornets, samt einer proportionirten Erböhung für die Stats Personen, und die Unterofficierer, wann Sie aufs genaueste, und gleichsam nur mit einem Wartegeld tractiret werden sollten, zum wenigsten monathlich $4000 \mathrm{Thl}$. thut alßo des Jabrß $300000 \mathrm{Thl}$. wofern nun das Fürstl. Hauß den vierdten Theil (salvo calculo) als 75000 Thl. beÿ truge, [fol. 158r] bliebe auf Ihrer Churfurstl. Durchl. und der Herrn Brüdere Landen 225000 Thlr. darauß folget, daß noch 8 oder 9 Quatember, und alßo über die bißherige Extraordinaria noch weit mehr, alß ein $\beta$ so viel angelegt, werden müste, wann gleich in diesem Quanto das Kre $\ddot{\beta}=$ Contingent mit begrifen seyn sollte.

20.

Were müglich, Ibre Churfürstl. Durchl. zubewegen, daß Sie von andern allbereit auf des Landes Kosten, aber in dero eigenen Bezablung habenden Garden und Völkern ein ziemliches in die vorberstehende Anzahl mit rechnen ließen, so würde zwar die Last umb viel erleichtert, dennoch aber überschwehr und groß verbleiben, und vernimmet mann zwar solche Discuss, mann sollte die II Soldaten in die Dörfer außtheilen, es würde kaum ein II Mann auf eines kommen, [fol. 158v] item mann sollte Ibren zum Theil Commiß oder Portiones geben, es scheinet aber, daß der erste calculy auf die Dörfer nicht ander $\beta$ anginge, alß wann die Leute sonst nichts oder wenig geben, wann Sie aber Monathlich zweÿ oder mehr Abrichtungen an Land= Trank= Fleisch Stewer, Quatember und Erbgefällen haben, so ists eben so schwebr den Soldaten im Dorf, alß in der Garnison zuerhalten geben auch vielmehr Insolentien vor, und ist die Disciplin und Exercitium vielschwehrer: mit dem Commiß aber wird es zwar in etwas aber nicht viel 
erleichtern, und weil obne dem der Unterthan zu Abstattung seiner Gefälle alles zu Geld machen muß, und nichts erspabren kan, so wird Ihn ein weniges an Getreide eher ins Commi $\beta=A m t$, al $\beta$ zum Markt schafen kann, muß aber doch hingegen so viel entbebren. [fol. 159r]

21.

Wo aber dergleichen hohe Summ und Mittel, wie es allem Ansehen nach, die Noth selbst alßo, wo nicht ietzo, doch in kurtzer Zeit lebren wird, nicht aufzubringen, II so würde auf Wartgelder zu gedenken seÿn, und II alßo den Gemeinen etwan balber Sold gegeben werden müßen, denn tüchtige Officirer werden sich nicht gar zu gering tractiren laßen, es müsten aber gleichwohl diese Wartgelder zum wenigsten wechßel weiße von Quartalen zu Quartalen, oder wie mann die Zeit eintheilete, zusammen gefordert, exerciret, und commandiret werden, wie Kriegsmanier ist. Wollte mann auch noch ein mebrers erspabren, so müste mann die Pferde von den Wartgelds Reutern nicht halten laßen, weil auf den Nothfall allezeit Pferde im Lande vorhanden, [fol. 159v] auf eine Caßa zum Pferdekauf gedenken, und nur Sattel, Zeng, Carbiner und Pistolen in Vorrath haben.

22.

Die andere gemeine Defensioner oder $A u \beta s c h u \beta=V e r f a ß u n g$ würde zwar nicht außer acht zulaßen seÿn, aber weil kein Exempel zu allegiren, daß damit jemabls etwas wichtiges außgerichtet, ein Feind abgebalten, oder ein Bündnuß darauf fundiret worden, so ists mehr zum Schein, als zu rechtem Nutzen, allenfalls aber (were) solche auf eine andere Art zu beratschlagen und anzustellen, alß bißhero geschehen, denn wie solche hiebevor im Churfürstenthum auf monathlichen Wartgeld bestanden, und vor wenig Jabren eingestellet worden, ist Sie allzu kostbar, und gleichwobl an der [fol. 160r] Mannschaft zu geringe, in andern Landen aber vielleicht allzu sebr ergrößert, dabero eine Mittelstraße rathsam seÿn möchte. Und weil das Volk auf dem Lande seine schwehre Last mit Akerwerk und Frohne hat, auch mit vielen Kosten und Unbequebmligkeiten zusammen zubringen, darbeÿ schlecht bekleidet, und geringes Vermögens ist, so were nach * dem Exempel der alten Vorfabren, die meiste und beste Defension auf die Bürgerschaft der Stätte zusetzen, denn * in denselben werden obne das sonst allerorten die Bürger mit dem Schießen exerciret, und haben Ihre Gewehr, nur daß es auf einerle ÿ Art und Loth einzurichten. Mann könte auch in den meisten Stätten durch Einziebung anderer wobl entbehrlicher Außgaben ein [fol. 160v] mehrers auf Besoldung guter Statt Officierer zum exercitio und dergleichen erspabren und anwenden, auch die Junge Pursche, wenn Sie gleich nicht Bürger, mit sonderlichen Satzungen, auch Ergetzlichkeiten obligiren, sich des Schießens und Exercirens beÿ Zeiten zugebrauchen. Auß diesen exercirten Bürgern könnte mann zur Bë̈hülfe der Geworbenen, und zu Besetzung der Plätze, eine gewiße Anzahl, reiheherumb, wie vor alters, kraft der Heerßfolge bescheben, heraus nebmen, so oft es nöthig, * maßen doch die neu geworbenen Knechte auch mebrentheils nichts alß Handwerks Pursche sind, * und ist zuermeßen, was nur der zebende oder zwantzigste Mann aus denen Stätten in den Chur= und Fürstlichen Landen außtragen würden, [fol. 161r] welche die Herrschaft nichts, oder ein weniges kosteten. Auf dem Lande könte mann bingegen die Anzabl mindern, und nur die jüngsten und burtigsten herauß lesen, auch die Knechte und Junge Pursche gleicher Gestalt mit herbeÿ ziehen, hingegen die Verheÿratheten, wann Sie über 40. Jabr weren, verschobnen (könne), und solches alles obne Wartgeld:, nur daß Officierer zum exercitio bestellet würden. Und weil es auch an tüchtigen Officierern zu Fuß gebrechen will, were wohl practicirlich, mit Rath einer Landschaft gewiße Mittel und Stiftungen zuerfinden, daß Junge von Adel und Bürgers Söhne mit zulanglichen Stipendÿs in fremde Lande geschiket würden, da Sie Kriegs Exercitia lerneten, 
und etwas redliches versucheten. [fol. 161v] So könten auch inner Landes etliche der leichtesten Artillerie Künste, alß Stükeschießen, Granaten werffen und dergleichen, wie etliche andere fremde Potentaten thun, mit einiger Mannschaft obne sonderbabren Kosten geübet werden, und in den Stätten nach und nach Verordnung geschehen, daß wieder etwas von Geschütz verschafet würde, wie vor alters gewesen.

\section{3.}

Es mögen nun auß vorherstehenden Mitteln viel oder wenig zu practiciren stehen, welches dann alles der genaue Uberschlag, und die vernünftige unpassionierte Betrachtung des Landes Zustand weißen wird, so were doch umb der Größe oder Geringfügigkeit der Mittel willen, die Vereinigung und Confoederation des gesamten Haußes nicht zu unterlaßen, theils daß mann dadurch be y Fremden eine Reputation [fol. 162r] erlanget, welche von Unßer innerlichen Beschaffenheit und Gebrechen nicht eben alle Nachricht haben, und sich davon vielleicht mehr einbilden, al $\mathrm{im}$ Werk zufinden, theils daß mann dadurch der Posterität consuliret, wo etwan Gott beßere Zeiten und Mittel beschebrete, oder die Noth noch größer würde, auf solchen Fuß sich weiter anzugreifen.

24.

Beÿ der Form und Administration solcher Zusammensetzung würde nun viel zubedenken stehen, weil alle bohe Interessenten sonder allen Zweifel gute Versicherung desiderirten, daß mann alles treulich meine, und bë̈ einander halten wolle, auch der darzu deputirten Mittel, und deren rechter und nützlichen Anwendung versichert së̈.

25.

Da dem zuförderst einander [fol. 162v] aufrichtig eröfnet werden müste, wie mann schobn gefast së̈ oder nicht, was mann an Volk, Ritterpferden, Defensionern habe, oder haben könne, wie mann mit Artillerie und Munition, auch einen Geld Vorrath oder Landesverwilligungen verseben, oder aufbringen könne, in was für Bündnüßen und Alliancen mann stehe, wie mann solche zu diesem Zwek appliciren, oder da es nicht müglich, mit guter Manier deren Relaxation, Declaration oder Milderung erbalten könne, welches desto eher geschehen wird, wo hingegen die Allÿrten mebrern Zugang oder Sicherheit von dem gantzen Hauße zugewarten, oder für deßen Offens sich zubesorgen hetten: und weil Gott nicht verboten, zuläßige Bündnüße zu Conservation des Seinigen einzugeben, so stünde mit höchster [fol. 163r] Vorsichtigkeit zuerwegen, welche nach Gelegenheit dieser Lande die besten und nützlichsten së̈en, ob und was mann von denen Allÿrten für Hülfe an Volk oder Geld haben könne, allermaßen sich tergteithen Mroßmächtige Reiche (dergleichen Mittel) zubedienen nicht entbrechen können, nur daß zum Fundament gesetzet würde, daß keiner von diesem Hauße einseitig und obne Wißen und Willen die Mitverwandten sich in Krieg oder Fried einließe, auch da gleich nur junge Herrschaften in Kriegsdiensten sich üben wollten, solches gleicher Gestalt communicato Consilio geschehe, damit Land und Leute nicht beschwebret, und in Gefahr gesetzet würden.

26.

$Z u$ richtiger Manutenenz geböreten nun auch, wie schon oberwebnet, sonderbabre verpflichtete [fol. 163v] Räthe, die mann in solchen wichtigen Dingen vertraulich brauchen, zusamnenschiken, Proviant, Munition oder Geld Vorrath durch dieselben aufbringen, auch in gesamt verwabren, und in Gewißheit obne Eingrif halten könte, darzu weren nun nicht nur Diener beÿ den Höfen, sondern auch etliche tapfere Leute auß der Landschaft von Ritterschaft und Stätten zugebrauchen, damit diese schwebre Last desto williger getragen würde, wenn mann deren rechte und getreue Administration in so vieler ehrlichen und verpflichteten Patrioten Händen wüste. 
27.

Die hohen Principalen selbst würden unter sich einen Modum pacisciren, wie Sie mit den allerwenigsten Kosten zusammen kommen, deliberiren und schließen könten, wie die Communicationes von Churfürstl. Durchl. an die [fol. 164r] andern Herren Interessenten unterschiedlich ergehen, und die Bedenken und Vota beförderlich eingehoblet werden könten, es möchte auch wobl dienlich und fast nöthig së̈n, daß im Nabmen aller der andern * Herrn Interessenten ein oder mebr tüchtige Subjecta am Churfürstlichen Hof residireten, mit denen mann continué communiciren, und durch die * selbe die Consilia zusammen bringen könte, denn mann bißhero genugsam verspühret, was auch gantz außwürdische Potentaten durch dergleichen Mittel an den Höfen gewirket. In Summa, wenn mann nechst Gott und seiner Ebre, bedenken will, was der hoben Obrigkeit höchste Pflicht und wabrer Nutz së̈, nebmlich nicht nur in bohem Stande nach Lust und Willen zuleben, sondern Seine [fol. 164v] von Gott anvertraute Land und Leute in Friede und Gerechtigkeit zuregiren, und dieselbe beÿ immerwebrender Nabrung und Aufnebmen, zu der Herrschaft eigenen Besten zuerhalten, in Betrachtung, daß Sie von denen Unterthanen Ibren Unterhalt nebmen muß, und alßo deren Woblfabrt mit der Ibrigen unaufläßlich verbunden ist, So kann mann noch wobl Mittel und Wege finden, dem Unheil, wo nicht auf einmabl, doch nach und nach zustenern, Im wiedrigen, und * da mann be ÿ dem unerschwinglichen Aufgang der Höfe bestehen, nichts zu des Landes besten erspabren, * und alles, was nur in Vorschlag kommet, von dem Schweiß und Blut der Unterthanen erwartten und haben will, und die schon am Tag [fol. 165r] stebende eußerste Noth uns von solchen eiteln Gedanken nicht abfübret, so wird alle menschliche Weißheit und Berathschlagung, auch Zusammensetzung und Verfaßung nicht gedej̈en, sondern von redlichen und frommen Hertzen nach und nach von allen Rathschlägen abstrabiret, und in Geduld erwartet werden müßen, was Gott verhengen wird. 